\title{
Screening of methicillin-resistant Staphylococcus aureus colonization at the admission: general surgical versus gynecological intensive care unit
}

\author{
1. Author: Krešimir Reiner, MD . Affiliation: resident in anesthesiology and intensive care medicine at the \\ Department for obstetrics and gynecology at the University Clinical Hospital Center Zagreb; Petrova 13, \\ 10000 Zagreb \\ 2. Author: Sandra Fudurić, MD. Affiliation: specialist in anesthesiology and intensive care medicine at the \\ Department for obstetrics and gynecology at the University Clinical Hospital Center Zagreb; Petrova 13, \\ 10000 Zagreb \\ 3. Author: Ljiljana Mihaljević, MD, Phd. Affiliation: specialist in anesthesiology and intensive care medicine \\ at the Department for obstetrics and gynecology at the University Clinical Hospital Center Zagreb; Petrova \\ 13, 10000 Zagreb \\ 4. Author: Slobodan Mihaljević, MD, Phd. Affiliation: specialist in anesthesiology and intensive care \\ medicine at the Department for obstetrics and gynecology at the University Clinical Hospital Center Zagreb; \\ Petrova 13, 10000 Zagreb
}

Background and goal of the study

Detection of methicillin-resistant Staphylococcus aureus (MRSA) colonization of nasal vestibule, oropharynx and perineum by taking swabs at the admission to the intensive care unit (ICU) has a great significance in reducing morbidity and mortality, since the colonization is early detected, which allows earlier patient isolation and application of procedures for MRSA eradication. Due to our clinical observations, we decided to compare the frequency of MRSA colonization between the two ICUs in the same hospital: general surgery ICU and gynecology ICU.

\footnotetext{
Materials and methods

Approval from the Ethical Committe of the University Clinical Hospital Center Zagreb was obtained. We included patients scheduled for major elective general surgery and gynecology procedures. Patients were divided into two groups by the type of ICU at which they were administered immediately after the procedure: general surgery ICU (group 1) and gynecology ICU (group 2). The two ICUs are located in the separate buildings of the same hospital. Operating rooms and ICU environment do not differ between the two groups, as well as the hygiene protocols among the hospital personnel. From the general surgery ICU, total of 161 patients were included in the study. From the gynecological ICU, we included total of 40 patients till present. At the admission to the ICU, we took swabs of nasal vestibule, oropharynx and perineum in each patient. Diagnosis of MRSA colonization was established by the microbiological analysis.
}

Results and discussion

It is often hard to discern the difference between colonization and infection. The swabs were taken immediately upon completed surgical procedure. Accordingly, all of the positive findings were cases of colonization. Results are shown in table 1 .

\begin{tabular}{|l|l|l|l|l|}
\hline & Group 1 & Group 2 & $\mathbf{X}^{2}$ & $\mathbf{P}$ \\
\hline $\begin{array}{l}\text { Nasal } \\
\text { vestibule } \\
\text { colonization } \\
\%\end{array}$ & 4.35 & 0 & 0.741 & 0.389 \\
\hline $\begin{array}{l}\text { Oropharynx } \\
\text { colonization } \\
\%\end{array}$ & 5.59 & 0 & 1.261 & 0.27 \\
\hline $\begin{array}{l}\text { Perineum } \\
\text { colonization } \\
\%\end{array}$ & 2.48 & 0 & 0.139 & 0.709 \\
\hline
\end{tabular}

\section{Conclusion}

There is no statistically significant difference in colonization between the two groups. However, cases of colonization were found exclusively among general surgical ICU patients till present. Since the study is ongoing, the final data analysis will show if there really is a difference, considering MRSA colonization, between the two groups. If present, the reasons for the difference are also to determine. Rates of MRSA colonization among general surgical ICU patients are satisfying when compared with other centers. 